Aus der Universitäts-Poliklinik für Ohren-, Nasen- und Kehlkopfkrankheiten in Breslau.

\title{
Zur Entstehung der otitischen Kleinhirnabszesse: Infektion durch den Hiatus subarcuatus.
}

Von Prof. Dr. V. Hinsberg.

Der Ohrenarzt ist häufig in der schwierigen Lage, gegen eine intrakranielle Komplikation der Otitis media operativ vorgehen zu müssen, deren Vorhandensein sich auf Grund der klinischen Symptome zwar vermuten, deren Charakter und Sitz sich aber nicht einmal mit Wahrscheinlichkeit vorher bestimmen läßt. Die Gründe dafür sind wohl genügend bekannt: je früher der Herd im Schädelinnern aufgedeckt wird, um so günstiger sind die Aussichten auf Erfolg; wenn man wartet, bis sich die Diagnose eines Hirnabszesses oder einer Meningitis erst mit Sicherheit stellen läßt, ist es oft für die Therapie zu spät. Man wird deshalb in verdächtigen Fällen am besten so verfahren, daß man den primären Eiterherd in Mittelohr und Warzenfortsatz operativ freilegt und dabei sorgfältig darauf achtet, ob der anatomische Befund irgend welche Anhaltspunkte dafür liefert, ob und in welcher Richtung. der Prozeß nach dem Cavum cranii zu weiter fortgeschritten ist.

Zur Beurteilung dieser Verhältnisse ist natürlich genaueste Kenntnis der Wege, die hierfür überhaupt in Frage kommen, notwendig. Diese sind heute durch die überaus zahlreichen Autopsien in vivo, die bei Operationen gemacht wurden, und durch sorgfältige Sektionen genau bekannt. Wenn ich sie kurz charakterisieren darf, so ist in einem Teil der Fälle die Eintrittspforte für das Infektionsmaterial aus den Mittelohrräumen ins Schädelinnere erst durch pathologische Vorgänge geschaffen worden: der schützende Knochenwall zwischen beiden wurde durch Einschmelzungsprozesse, Frakturen oder ähnliche Vorgänge zerstört. In einer Minderzahl von Beobachtungen fand man, daß das entzündliche Agens zu seiner Invasion ins Cavum cranii präformierte Bahnen benutzte. Was diesen zweiten Infektionsmodus betrifft, so erfolgt wohl am häufigsten die Ausbreitung vom eitrig erkrankten Labyrinth aus, entlang den Nerven, viel seltener durch die Aquädukte. Zu den sehr seltenen Wegen gehören Dehiszenzen am Dach oder Boden der Paukenhöhle, die Canaliculi carotico-tympanici, die Fissura petro-squamosa, der Facialiskanal und endlich der Hiatus subarcuatus. Während sich die pathologischen Knochenlücken bei sorgfältigem Suchen während der 
Operation meist auffinden lassen und uns auf die richtige Spur leiten, lassen sich Infektionen durch die präformierten Bahnen so gut wie niemals makroskopisch erkennen. Um so wichtiger ist es deshalb, daß man die Möglichkeit ihres Vorkommens genau kenñt, um eventuell auch ohne Wegeleitung den intrakraniellen Herd auffinden zu können. Von diesem Gesichtspunkte aus scheint mir die Mitteilung der nachfolgenden Beobachtungen wünschenswert, da sie uns einen Modus für die Entstehung eitriger Prozesse in der Kleinhirngrube illustriert, der bisher nur wenig bekannt war.

Bei fast sämtlichen bisher beschriebenen Fällen von Kleinhirnabszessen nämlich, bei denen der Infektionsmechanismus überhaupt festgestellt werden konnte, war die Eiterung entweder vom Labyrinth aus oder durch Vermittlung des Sinus sigmoideus, beziehungsweise seiner Umgebung aufs Cerebellum übergegangen, so daß Koch in seiner Monographie ${ }^{1}$ ) über den Kleinhirnabszeß den Satz aufstellen konnte: „Es gibt zwei Quellen für den Kleinhirnabszeß von gleicher Bedeutung: das Ohrlabyrinth mit dem angrenzenden Teil des Antrum mastoideum und den Sulcus mit dem Sinus sigmoideus." Zum gleichen Resultat kam $0 \mathrm{kada},{ }^{2}$ ) der unter 109 Kleinhirnabszessen in $95,5 \%$ eine Labyrinth-Eiterung oder Sinusphlebitis, beziehungsweise Periphlebitis nachweisen konnte, während nur bei $4,5 \%$ das Felsenbein und der Sinus anscheinend gesund waren.

Man war'also auf Grund dieser Erfahrungen berechtigt, einen Zerebellarabszeb zum mindesten für sehr unwahrscheinlich zu halten, wenn Labyrinth und Sinus sich als intakt erwiesen. Auch wir waren bis zur Beobachtung der nachfolgenden beiden Fälle dieser Ansicht, die wir jedoch unter Berücksichtigung früherer, gleich zu erwähnender Erfahrungen dahin modifizieren müssen, daß neben den beiden Hauptinfektionspforten für die Kleinhirngrube als dritte der Hiatus subarcuatus eine nicht zu vernachlässigende Bedeutung besitzt.

Whe ich auf unsere Fälle näher eingehe, sei es mir gestattet, mit wenig Worten das Wissenswerte über den Hiatus subarcuatus, der wohl nur dem Anatomen und Otologen von Fach genauler bekannt sein dürfte, zu sagen. ${ }^{3}$ )

Bei einer großen Reihe von Säugetieren befindet sich unter dem oberen Bogengang eine rundliche Oeffnung, die lateralwärts in einen mehr oder weniger großen Knochenhohlraum führt: die Fossa subarcuata. Sie ist von Dura ausgekleidet, und in ihr liegt meist ein Läppchen des Kleinhirns. Während diese Grube bei einigen Affen und allen übrigen Säugetieren, die sie überhaupt haben, während des ganzen Lebens persistiert, ist sie bei dem andern Teil der Affen und dem Menschen nur beim Neugeborenen vorhanden, im Laufe der extrauterinen Entwicklung bildet sie sich jedoch in der Weise zurück, daf die Grube ganz von Knochen ausgefüllt wird. Es bleibt an ihrer Stelle, also etwas medial von der durch den oberen Bogengang gebildeten Eminentia arcuata, nur eine leichte Einziehung übrig. Von dieser aus zieht, wie man auf Serienschnitten durchs Schläfenbein deutlich verfolgen kann, in einem feinen Knochenkanal ein in Bindegewebe eingebetteter Gefäßstrang, der die Schleimhaut der hinteren oberen Warzenfortsatzzellen mit der Dura in der Gegend der eingezogenen Stelle verbindet. Auch an Metallkorrosionspräparaten vom Schläfenbein ist der Gefäßstrang meist sichtbar. Nach Ansicht einiger Autoren soll er mit dem Sinus petrosus superior zusammenhängen. Dieser Kanal mit seinem Inhalt ist das, was man gewöhnlich als Hiatus subarcuatus bezeichnet.

Wie oben erwähnt, sind einige Fälle, bei denen der Hiatus bei der Fortleitung von Mittelohreiterungen aufs Schädelinnere eine Rolle gespielt hat, bereits publiziert. Bei den meisten war jedoch bei der Sektion der Zusammenhang zwischen Hiatus und Hirnerkrankung nicht mehr ganz klar zu rekonstruieren, wie aus den nachfolgenden Auszügen hervorgeht.

1. Beobachtung von Voltolin $i^{+}$). 27jähriger Mann, gestorben an Meningitis und Kleinhirnrindenabszeß. Die Infektion war entweder vom Labyrinth, das vereitert war, oder vom rechten Hiatus aus erfolgt. „Die Stelle, wo im normalen Zustande unter dem Bogen des Canalis sup. durch die Knochenmasse eine Oeffnung mit einer Falte der Dura geht, die sonst sehr klein ist, zeigte hier eine sehr große Oeffnung, die von einer blutig-roten Masse angefüllt war."

1) Koch, Der otitische Kleinhirnabszeß. Berlin 1897. - 2) Okada, Diagnose und Chirurgie der otogenen Kleinhirnabszesse. Jena 1900 . - 3) Eine ausführliche Beschreibung gibt Wagenhäuser in seinen "Beiträgen zur Anatomie des kindlichen Schläfe beins". Archiv für Ohrenheilkunde Bd. 19. - 4) Voltolini, Virchows Archiv Bd. 31 .
2. Beobachtung von v. Tröltsch'). 20jähriges Mädchen, das an linksseitiger chronischer Otorrhoe litt. Gestorben an Kleinhirnabszeß und Schläfenlappenabszeß. Bei der Sektion fand sich eine Labyrintheiterung, der Porus ac. int. war frei von Eiter. Außerdem bestand eine Phlebitis des Sinus petr. sup., die nach v. Tröltschs Ansicht vielleicht durch den Hiatus subarcuatus fortgeleitet war. In diesem zog „ein zirka $1 \mathrm{~mm}$ dicker, hier ganz geröteter Gefäßstrang unter dem Scheitel des Canal. semic. sup. durch den Knochen nach hinten und außen zum Antrum mastoideum".

3. Beobachtung von $\mathrm{H} a r t \operatorname{mann}^{2}$ ). 37jähriger Mann, chronische Otitis media linkerseits. Caries des Felsenbeins, Eiterdurchbruch nach der hinteren Schädelgrube. Meningitis purulenta. Kleinhirnabszess. Sektionsbefund: Knöcherne Wand des Facialiskanals an seiner Umbiegungsstelle vollständig zerstört. Von hier führen zwei kariöse Gänge nach hinten, beide von der mittleren Schädelgrube durch eine dünne Knochenplatte getrennt. Der eine Gang entspricht dem Hiatus subarcuatus, an seiner Mündung befinden sich kleine periostitische Auflagerungen. Genaueres über das Verhältnis des walnußgroßen Abszesses zum Hiatus konnte Hartmann, der nur das schlecht konservierte Schläfenbein zur Untersuchung hatte, nicht mehr feststellen.

4. Beobachtung von $\left.\mathrm{Kü} \mathrm{m} \mathrm{mel}{ }^{3}\right)$. 36jähriger Mann, geheilte, an scheinend rezidjvierende Otitis media links, Mastoiditis. Großer extraduraler Abszeß und Schläfenlappenabszeß. Eröffnung und Entleerung beider. Tod an Pneumonie. Bei der Sektion fand sich eine unter Bindegewebsneubildung ausgeheilte Labyrinth-Eiterung. An der oberen Pyramidenkante, dicht hinter und medialwärts von der Eminentia arcuata, eine zirka linsengroße, gelblichweiß verfärbte Partie des Knochens, von ganz feinen Kanälchen siebartig durchbrochen. Diese sind von offenbar eiterartiger Masse erfüllt. Die betreffende Knochenpartie grenzt dicht an eine zwischen oberen und lateralen Bogengang sich eindrängende Ausbuchtung des Antrum mastoideum, die sich bei der mikroskopischen Untersuchung als mit Granulationen ausgefüllt erweist. Der Knochen ist von Rundzellen reichlich durchsetzt, anscheinend nekrotisch. Von der Ausbuchtung zieht zur siebartigen Stelle ein zirka $1 \mathrm{~mm}$ starker, gefäßhaltiger Bindegewebsstreifen, stark kleinzellig infiltriert, der Knochen in seiner Umgebung ist schlecht färbbar, rundzellenreich. Der Verlauf des Stranges stimmt nach $\mathrm{K}$ ü m m els Ansicht recht gut mit dem des Hiatus subarcuatus überein, und $\mathrm{Kümmel} \mathrm{hält}$ es für möglich, daß durch ihn eine früher vorhandene Otitis media auf das Labyrinth und gleichzeitig oder später auf die Schädelhöhle übermittelt wurde. Als zweite Möglichkeit erwähnt er, daß nicht eine Otitis media das primäre war, sondern daß ein primärer osteomyelitischer Herd in der Umgebung des subarkualen Bindegewebsstranges (der Patient hatte früher eine Osteomyelitis der Tibia durchgemacht) die Ursache für die Mittelohreiterung sowohl wie für die Infektion des Cavum cranii abgegeben habe.

5. Beobachtung von $\mathrm{Muck}^{4}$ ) aus der Körnerschen Klinik. 15jähriger Knabe, chronische Otitis media sinistra, Leptomeningitis und Extraduralabszeß 3 in der hinteren Schädelgrube. Granulationen auf dem Sinus ohne Veränderungen an dessen Intima. Operation, Tod. Sektionsbefund: Eiter in Vorhof und Bogengängen, Schnecke frei. Steigbügelplatte intakt. Por. ac. int. frei von Eiter. Saccus endolymphaticus mißfarbig, seine beiden Blätter teilweise ulzeriert. Von einer Höhle an der inneren Antrumwand führt eine Oeffnung direkt in den Hiatus subarcuatus. Bei der Sektion ließ sich nicht mehr genau feststellen, ob die Meningitis vom Hiatus subarcuatus oder vom Saccus endolymphaticus ausging. Auch auf welche Weise das Labyrinth infiziert wurde, war anscheinend nicht mehr zu eruieren.

Endlich sei noch ein Fall von Odenius erwahnt, den v. Trölts $\mathrm{ch}^{5}$ ) kurz zitiert, der mir jedoch nicht zugänglich war.

Klarer als in den oben zitierten Mitteilungen lagen die Verhältnisse in folgendem, an der Breslauer Poliklinik beobachtetem Fall.

Stanislaus K., ein zirka 40jähriger Arbeiter, litt seit zirka zwei Jahren an linksseitiger Mittelohreiterung. Seit zwei Tagen plötzlich wütende Schmerzen im linken Ohr, angeblich, nachdem ein Strohhalm hineingeraten war. Dabei mäßiger Ausfluß, Fieber, Schmerzen im Hinterkopf.

Aufnahmebefund am 23. Juli 1901: Patient hat ohne Unterstützung dic Reise aus der mehrere Stunden entfernten Heimat nach Breslau gemacht und, soweit mir erinnerlich (Notizen darüber fehlen in der Krankengeschichte), zu Fuß oder mit Benutzung der Trambahn die Klinik erreicht. Er macht einen sehr matten Eindruck. Die Gesichtsfarbe zeigt nichts Auffallendes, das Sensorium ist frei. Temperatur 38,2 , Puls 65 . Hörprüfungsresultat nicht notiert. Zurzeit kein Schwindel. Infiltration über dem Proc. mastoid. Im Gehörgang krümeliger Eiter. Totaldefekt des Trommelfells.

24. Juli morgens Totalaufmeißelung in ungestörter Narkose. Proc. mastoid. sehr markreich, in den Hohlräumen kein Eiter. Bei der Eröfnung des Antrums entleert sich eine Menge trüber Flüssigkeit. Im

1) v. Tröltsch, Archiv für Ohrenheilkunde Bd. 4. - 2) Hartmann, Zeitschrift für Ohrenheilkunde Bd. 8. - 3) $\mathrm{K}$ ü m me l, Zeitschrift für Ohrenheilkunde Bd. 31. - 4) $\mathrm{Muck}$, Zeitschrift für Ohrenheilkunde Bd. 37. - 5) l. c. 
Antrum grauweißliche, stinkende Massen, die sich auch in die Paukenhöhle erstrecken. Der freigelegte Sinus sieht gesund aus und pulsiert. Körnersche Plastik, Tamponade, Verband.

Nach dem Erwachen aus der Narkose klagt Patient über heftige Hinterkopfschmerzen. Nachmittags gegen 4 Uhr plötzlich Temperaturanstieg von $36,8^{\circ}$ auf $41,2^{\circ}$ innerhalb einer Stunde, gleichzeitig Anstieg der Pulsfrequenz, die vormittags 70 betragen hatte, auf ungefähr 200 . Völlige Bewußtlosigkeit. Links Patellarreflex gesteigert, rechts nicht. Rechte Pupille maximal eng, linke mittelweit. Rechts anscheinend Facialisparese. Cheyne-Stokessches Atmen. Abends $7 \mathrm{Uhr}$, also drei Stunden nach Beginn der bedrohlichen Symptome, Exitus.

Sektionsbefund: Am linken Por. ac. int. bohnengroße Verklebung zwischen Cerebellum und Pyramidenoberfläche. Unterseite der linken Kleinhirnhemisphäre von eitrigen Auflagerungen, die zum Teil schwartige Beschaffenheit angenommen haben, bedeckt. In der linken Hemisphäre des Cerebellums ein etwa kirschgroßer, in zwei Kammern geteilter Abszeß. Hirnstiele, Brücke, Optici graugelb belegt. Uebrige Organe ohne Befund. Das linke Schläfenbein wird herausgenommen und in Serienschnitte zerlegt. Schnittrichtung: senkrecht zur Pyramidenkante und parallel zur Schneckenachse.

Mikroskopischer Befund: Die Schleimhaut der medialen Paukenhöhlenwand ist mächtig verdickt, kleinzellig infiltriert. Steigbügelplatte und Membrana tympani secundaria intakt. Labyrinth ohne pathologische Veränderungen. Acusticus im Meat. audit. int. kleinzellig infiltriert (sekundäre Infiltration durch Fortkriechen der Meningitis). Antrum leer, seine Wand stellenweise von Granulationspolstern bedeckt. Ein solches von ziemlich erheblichem Umfang findet sich im hinteren Pol des Antrums. Von hier aus geht der Hiatus subarcuatus, der sich als ein ovaler, zunächst gerade verlaufender Kanal von zirka $1 \mathrm{~mm}$ Höhe und $2 \mathrm{~mm}$ Breite durch den Knochen bis zum oberen Bogengang verfolgen läßt (Fig. 1). Medial von diesem biegt

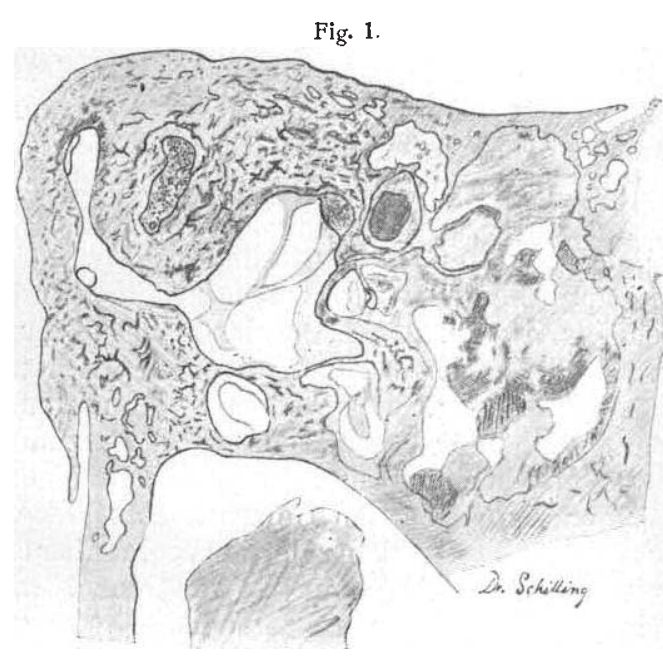
er rechtwinklig um und mündet mittels einer weiten Oeffnung an der hinteren Pyramidenfläche, dicht unterhalb derKante. Der Kanal, dessen Wandungen völlig glatt sind und keinerle Zeichen von Nekrose zeigen, enthält eine Anzahl von Gefäßen Das größte von ihnen eine Arterie, ist erheblich verändert: die Adventitia ist stark gewuchert, die Intima verdickt, in Falten gelegt. Das Lumen ist dadurch sehr verengt, aber nicht thrombo-

siert. Die Gefäße sind eingebettet in ein außerordentlich zellreiches Granulationsgewebe, das mit dem beschriebenen Polster im Antrum kontinuierlich zusammenhängt und sich bis zur Mündung des Hiatus an der hinteren Pyramidenkante verfolgen läßt.

Ein Zweifel über den Zusammenhang zwischen Mittelohreiterung und Hirnabszeb ist bei dem beschriebenen Befunde nicht möglich. Während einerseits eine andere Entstehungsweise für den Absze日 (Sinusthrombose, Labyrinth-Eiterung) mit Sicherheit ausgeschlossen werden kann, zeigt andererseits der Hiatus deutliche Veränderungen, so daß sich der Weg der Infektion vom Antrum bis zum Kleinhirn direkt unter dem Mikroskop verfolgen läßt.

Unser Fall zeigt hinsichtlich der Veränderungen im Hiatus große Aehnlichkeit mit dem oben erwähnten, von Kümmel beschriebenen, nur war der Knochen in der Umgebung des Kanals, der dort nekrotisch war, unverändert. Daß der Abszeß vor der Operation nicht diagnostiziert wurde, dürfte bei dem Fehlen aller charakteristischen Symptome - nur die Verminderung der Pulsfrequenz war verdächtig - verzeihlich erscheinen.

Während sich, wie schon erwähnt, bei diesem Fall das Fortkriechen der Eiterung im Hiatus mit aller Sicherheit nachweisen ließ, liegen beim folgenden die Verhältnisse etwas komplizierter.

Die 13jährige Frida T. trat am 10. Juli 1900 in die Behandlung der Poliklinik. Anamnestisch beiderseits chronische Otitis media, vor vier Wochen unter Frostgefühl akutes Aufflackern der Eiterung. Seit zwei Tagen heftige Kopfschmerzen, wiederholtes Erbrechen, Stechen und Summen in den Ohren. Aufnahmebefund: Sehr heruntergekommenes Kind von fahler Gesichtsfarbe, das vor Schwäche kaum stehen und gehen kann und dabei über Schwindelgefühl klagt. Brechneigung. Puls klein, 120 in der Minute, Temperatur $38,2^{\circ}$, keine Nackenstarre.
Leichte Ptosis links. Strabismus divergens. Keine Doppelbilder, keine Gesichtsfelddefekte, Augenhintergrund frei. Beiderseits fötider Eiter im Gehörgang, großer Trommelfelldefekt, Paukenhöhlenschleimhaut granulierend. Weber nach links lateralisiert, Töne der Galtonpfeife werden gut gehört, tiefe Töne schlecht, besonders links. Sprachgehör nicht notiert.

Auf Grund dieses Befundes wird ein intrakranieller Herd angenommen, doch läßt sich nicht einmal feststellen, ob er vom rechten oder vom linken $\mathrm{Ohr}$ ausgeht.

Am 11. Juli in ungestörter Narkose zunächst Totalaufmeißelung links. Sinus stark vorgelagert, seine Wand, die weit freigelegt wird, normal, ebenso die Dura der mittleren Schädelgrube. Warzenfortsatzzellen klein, zum Teil durch geschwollene Schleimhaut ausgefüllt, nirgends Eiter. In Antrum und Paukenhöhle Granulationen. Körnersche Plastik, Tamponade.

Da der Befund das schwere Krankheitsbild nicht erklärt, wird sofort die Operation der anderen Seite angeschlossen. Befund ganz analog, nur ist das Antrum nach hinten vergrößert und von weißen Massen ausgefüllt. Auch hier sieht die Dura über dem Schläfenlappen und dem Sinus normal aus. Plastik, Tamponade.

Während der nächsten Tage keine Besserung. Die Temperatur steigt vielmehr am Tage nach der Operation auf $39,2^{\circ}$, die Pulsfrequenz ist andauernd hoch. Die Ptosis links nimmt zu, zuweilen ist eine gewisse Somnolenz zu konstatieren. Leichter Nystagmus in beiden Endstellungen. Augenhintergrund normal. Erbrechen. Ueber Schwindel wird nicht mehr geklagt.

Deshalb am 16. Juli zweite Operation, und zwar werden zunächst wiederholt Probepunktionen in den rechten Schläfenlappen ausgeführt, die jedoch negativ ausfallen. Dann Spaltung der Dura über dem rechten Schläfenlappen: Dura nicht verdickt, keine Verwachsungen mit der Pia, diese normal aussehend. Jodoformgaze-Tamponade zwischen Dura und Pia. Im Anschluß daran Punktion in den linken Schläfenlappen, ebenfalls negativ, und Spaltung der Dura ïber ihm. Diese ist stark verdickt und mit den weichen Hirnhäuten verwachsen. Kein Eiter. Tamponade wie rechts.

Auf Grund dieses Befundes wird eine zunächst noch lokalisierte Meningitis am linken Schläfenlappen angenommen. Eine Punktion ins Kleinhirn wird unterlassen, weil die Dura über dem Sinus und seiner Umgebung vollständig normal war, und weil wir auf Grund der Hörprüfung eine Labyrinth-Eiterung nicht für wahrscheinlich hielten, somit die beiden Wege, auf denen die Infektion in der Regel die hintere Schädelgrube erreicht, anscheinend nicht in Betracht kamen.

Verlauf nach der Operation: Ptosis und Nystagmus gehen zurück. Ophthalmoskopisch wird beiderseits Neuritis optica incipiens konstatiert. Linke Pupille weiter als rechte, beide reagieren prompt. Pulszahl und Temperatur, die zunächst nach der Operation sanken, steigen am zweiten Tage wieder. Allgemeinbefinden sehr wechselnd, bald große Unruhe, so daß Patientin sich schreiend im Bett herumwirft und durch ihr ungezogenes Wesen die Umgebung sehr quält, bald Somnolenz, bald freies Sensorium. Nahrungsaufnahme sehr gering. Beiderseits bildet sich an der Stelle der Dura-Inzision ein starker Hirnprolaps aus. Beim Verbandwechsel werden wiederholt Punktionen in den Schläfenlappen ausgeführt, stets negativ. Am 2. August Exitus, nachdem die Patientin schon am Tage vorher vollständig somnolent gewesen war

Kopfsektion: Beiderseits beträchtlicher Prolaps des Schläfenlappens an der Stelle der Inzision. Großhirnoberfläche trocken. Hirnsubstanz etwas weich. In der Gegend des linken Porus ac. int. ist das Kleinhirn mit der Pyramidenoberfläche leicht verwachsen. Die Dura zeigt hier eine kartenblattdünne, rötlichgelbe Auflagerung. Die Dura wird an dieser Stelle mit dem Knochen in Verbindung gelassen. Im übrigen zeigt die Felsenbeinoberfläche nichts Besonderes. Sinus ohne Veränderungen. Keine Meningitis. In der linken Kleinhirnhemisphäre ein schwappender Abszeß, bei dessen Inzision sich zirka zwei Eßlöffel voll stinkenden Eiters entleeren.

Mikroskopische Untersuchung des in Serien zerlegten linken Schläfenbeins: Tegmen tympani et antri fehlen (operativ entfernt). In der Umgebung des Defekts ist die Oberfläche der Pyramide arrodiert; über das Verhalten der Dura läßt sich nichts mehr eruieren, da sie bei der Sektion abgezogen wurde. In der Paukenhöhle geronnenes Exsudat, Granulationen und Knochensplitterchen. Die mediale Paukenhöhlenwand ist epidermisiert. An den Labyrinthfenstern kein Durchbruch. Der Hiatus subarcuatus ist deutlich ausgebildet und auf den Schnitten schon makroskopisch als eine runde Oeffnung von zirka $1 \mathrm{~mm}$ Durchmesser erkennbar. Mikroskopisch sieht man, daß er von ziemlich derbem Bindegewebe ausgefüllt ist, in dem mehrere Gefäße liegen Weder Bindegewebe noch Gefäße zeigen pathologische Veränderungen Die Verbindung des Kanals mit der Schleimhaut der Warzenfortsatzzellen ist nicht mehr nachweisbar, da diese fehlen (operativ zerstört). Medialwärts verläuft der Kanal ganz gerade bis zum Durchtritt unter dem oberen Bogengang; unmittelbar, nachdem er diesen passiert, biegt er rechtwinklig um und erreicht die hintere Pyramidenfläche, dicht an der oberen Kante. Die Dura, die hier noch im Präparat erhalten ist, ist in einem Bezirk von zirka $6-8 \mathrm{~mm}$. Durchmesser stark verdickt, sie stellt einen $\mathrm{W}^{\top}$ ulst von derben Bindegewebsmassen dar, der stark 
vaskularisiert ist und stellenweise kleinzellige Infiltrationen zeigt. Das Zentrum dieses Bezirks, zugleich die dickste Stelle, liegt gerade über der Mündung des Hiatus subarcuatus, und man kann in der Serie genau verfolgen, wie die Gefäße des Kanals sich unmittelbar in die Dura versenken. Die Oberfläche des Schläfenbeins ist an dieser Stelle arrodiert, und zwar am stärkstẹn über dem hinteren Schenkel des oberen Bogenganges. Die Zerstörung reicht hier so tief, daß seine äußere Wand stellenweise total verschwunden ist, an einer Stelle ist sogar die mediale Wand angenagt (Fig. 2). Der häutige Bogengang ist hier

Fig. 2.

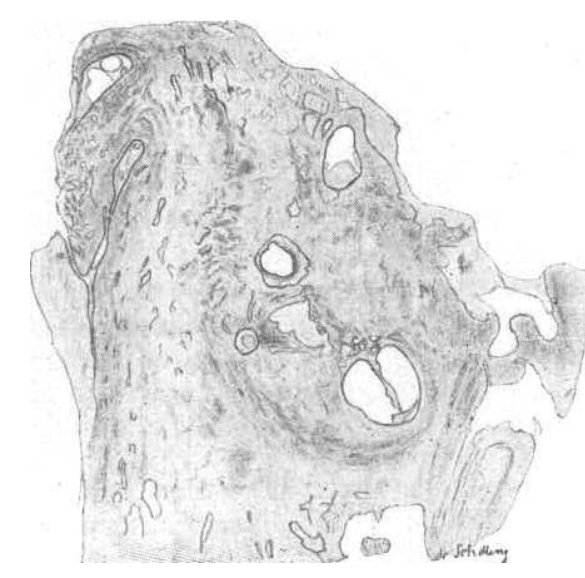
zugrunde gegangen, so daß die granulierende Dura der medialen Bogengangswand aufliegt. An der Grenze der Zerstörung sind an beiden Schenkeln die Wände des häutigen Bogengangs miteinander verklebt, sie zeigen hier geringe kleinzellige In. filtration. Im übrigen ist das Labyrinth frei von pathologischenVeränderungen; im perilymphatischen Raum sind stellenweise homogene Gerinnungsprodukte. Nervi acusticus und facialis ohne entzündliche Veränderungen. Bakterienfärbung gelingt nicht, offenbar, weil die Entkalkungsflüssigkeit zu lange eingewirkt hatte.

Der beschriebene Herd in der Dura entspricht der bei der Sektion erwähnten Auflagerung, und da gerade hier die Kleinhirnabszeßwand mit der Dura verwachsen war, ist er wohl zweifellos als der Ort anzusehen, an dem die Infektion ins Cerebellum eindrang.

Wie kam nun aber die Infektion gerade an dieser Stelle zustande? Das Labyrinth läßt sich als Vermittler bestimmt ausschließen, da in seinem Innern keinerlei entzündliche Prozesse nachzuweisen sind und da der Defekt am oberen Bogengang durch Arrosion von a $u B e n$ her bedingt ist und nicht etwa einen Durchbruch von innen nach außen darstellt. Kann man somit das Labyrinth als Infektionsträger ausschalten, so bleibt, da ein anderer Weg nicht $\mathrm{zu}$ sehen ist und ein direkter Zusammenhang zwischen Mittelohr und Abszel doch angenommen werden muf, nur der Hiatus übrig, dessen Gefäße ja auch, wie erwähnt, gerade ins Zentrum der betreffenden Stelle münden. Nun zeigen aber, im Gegensatz zu unserem ersten Fall, weder die Gefäße des Kanals noch das sie umgebende Bindegewebe deutliche pathologische Verhältnisse: erstere sind nicht thrombosiert, und letzteres ist nicht entzündlich verändert. Eine Infektion per continuitatem hat also sicher nicht stattgefunden. Wohl aber scheint mir die Annahme gerechtfertigt, daß durch die Gefäße infektiöses Material aus der Schleimhaut der erkrankten Warzenfortsatzzellen, mit der sie ja direkt zusammenhingen, in die Dura embolisch verschleppt wurde und daß sich hier ein entzündlicher Prozef etablierte, während am Hiatus selbst keine Spur dieses Vorganges zurückblieb.

Was die klinische Seite des Falles anlangt, so zeigt eine nachträgliche Ueberlegung, daß die Symptome, wenn sie auch keineswegs als charakteristisch bezeichnet werden können, doch allch an einen Kleinhirnabszeß denken lassen mußten. Es war entschieden falsch, daB wir uns durch das Fehlen jeder Wegeleitung zur hinteren Schädelgrube verleiten ließen, einen Zerbellarabszen für unwahrscheinlich zu halten. Erschwert wurde freilich die Beurteilung dadurch, daß sich bei der zweiten Operation im Bereich der mittleren Schädelgrube wirklich deutliche Veränderungen fanden, die für die Annahme einer Erkrankung im Bereich des Schläfenlappens eine objektive Grundlage lieferten. Wären diese nicht vorhanden gewesen, so würden wir zweifellos eine Probepunktion ins Kleinhirn ausgeführt haben.

Weitgehende Folgerungen aus dem bisher vorliegenden, spärlichen und zum Teil nicht ganz eindeutigen Material zu ziehen, ist nicht meine Absicht. Nur so viel wird man wohl heute schon schließen dürfen, daß die Infektion durch den Hiatus subarcuatus in der Mehrzahl der Fälle (fünf von den sieben angeführten) zur Entstehung von pathologischen Prozessen in der Kleinhirngrube allein führt, viel seltener zu analogen Veränderungen in der mittleren Schädelgrube allein oder in beiden Schädelgruben. $\mathrm{Ob}$ diese Verschiedenheit in der Ausbreitung der Infektion auf
Variationen in der Lage des Ausführungsganges des Hiatus zurückzuführen ist, etwa so, daß er in der Regel, wie bei unseren beiden Fällen, an der hinteren Pyramidenfläche liegt, in selteneren Fällen jedoch auf der Kante oder auf der vorderen Fläche, wage ich, da mir ausgedehnte Untersuchungen darüber nicht zur Verfügung stehen, nicht zu entscheiden, doch liegt eine solche Annahme wohl nahe.

Der Umstand, daß bei sechs von den angeführten sieben Fällen die Infektion durch den linken Hiatus erfolgt, nur einmal durch den rechten, dürfte wohl nur auf einen Zufall, der bei so kleinen Zahlen ja eine bedeutende Rolle spielt, zurückzuführen sein, ich kann mir wenigstens nicht vorstellen, wodurch in dieser Beziehung ein prinzipieller Unterschied zwischen links und rechts bedingt sein sollte. 